\title{
Light Path Optimization Implementation for Survivable Routing and Wavelength Assignment in Optical Networks
}

\author{
P. Rajesh M.Tech., Asst Prof ${ }^{1}$, G. Sree Hari M.Tech., Asst Prof ${ }^{2}$ \\ B. Mallikarjuna Naik M.Tech., Asst Prof ${ }^{3}$, \\ ${ }^{1,2,3}$ CRIT college of Engg, Anantapuramu.
}

\begin{abstract}
In this paper, establishing a light path setup in WDM network done by implementing an ant-based mechanism Ant Colony Optimization (ACO) algorithm used for providing survivable routing in WDM network. The major problem in survivable routing WDM network is routing wavelength and assignment (RWA) problem, It sets up light path by routing and assigning a wavelength to each connection such that no light path use the same wavelength on same link. Our study aims to develop an routing and optimize the light path setup for survivable routing based upon the bit transmission to which supports data transfer supports to run continuously thus it can provide dynamic path. By adopting a new routing table structure and keeping a number of mobile agents in the network to cooperatively explore the network states and continuously update the routing tables, our new ant algorithm can efficiently support the ants' foraging tasks of route selection and wavelength assignment in WDM networks. Each path in the network is assigned by feasibility value. This feasibility value is calculated by considering the metrics such as bandwidth availability, path length and number of free wavelengths. When an ant is launched in the network by its node, it moves towards random destination to gather information such as bandwidth availability, path length and free wavelengths in that path. The value of path load is dynamically updated by the mobile agents in the load table.

Using NS-2 simulation we show that the proposed ant based routing achieves better channel utilization and throughput with reduced delay, when compared with the normal routing. Optimization of light path performance is measured by varying the CBR traffic sending rate and flows.
\end{abstract}

Key words: WDM optical network, WDM light path, blocking probabability, delay, CBR, Expo

\section{Introduction}

In all optical networks, data are routed in optical channels called light paths. [1]The Routing and Wavelength Assignment (RWA) problem is how to determine both a route and wavelengths for a connection request. Without wavelength conversion capability, a light path must use the same wavelength on all the links along its route, which is referred to as the wavelength continuity constraint. In a wavelength-routed network, a connection between a source node and a destination node is called a lightpath. A lightpath is an optical channel that may span multiple fiber links to provide an all-optical connection between two nodes. In the absence of wavelength converters, a lightpath would occupy the same wavelength on all fiber links that it traverses. Two light paths on a fiber link must be on different wavelength channels to prevent the interference of the optical signals.

\section{Roadmap of Research}

This work addresses more detailed in optimizing light path approaches for allocating lightpath RWA and measuring of data bit rate transmission across each lightpath establishment and wavelength assignment. In previous work [3], Two basic algorithms, are .Ant based control (ABC) for telephone networks Schoonderwoerd et al.proposed an and Ant Net for packet switching networks, which was proposed by DiCaroetal.[4]. Some subsequent enhancement schemes to improve the ant-based routing performance include smart agents which use dynamic programming [5], Rajesh Rai . [6] proposed an ACO-based algorithm to solve the survivable RWA problem. When a new connection request arrives, a number of ants are launched from the source to the destination. Ants evaluate a path based on its length and the mean available wavelengths along the path.

Apart from this, we have more works for survivable routing in WDM networks. All these works generate backup paths using basic scheduling and routing mechanisms. In addition to this, the backup paths created by these mechanisms are static paths, which are unaware of impairments in links. The typical constraints for wavelength allocation problem are the limited wavelength number on each link and the wavelength continuity constraint for all WDM networks. The interconnected multilayer-graph model (IMG) [20] is used for the wavelength allocation. We consider that part of the nodes have wavelength conversion. For nodes without wavelength conversion capability, the wavelength continuity constraint is checked while establishing the light 
paths. The wavelength assignment algorithm assigns wavelengths to the primary and backup paths in order to share the resources between the current traffic requests and the already established requests.

\section{Optimizing Lightpath Routing And Wavelength Assignment}

Ant Colony Optimization: is a set of algorithms used for optimization of several problems.ACO algorithms try to enumerate biological behavior of ant foraging for food. In this section, the proposed ant based RWA algorithms, Ant Colony Routing and Wavelength assignment (ACRWA)is described in details. The main purpose of ACRWA is to run globally distributed optical wavelength assignment. ACRWA is based on well known Ant colony system (ACS) algorithm is a solution for resolving problem such as travelling salesman problem (TSP).RWA chief parameters solutions are blocking probability and number of wavelengths used.

\section{A) Lightpath Establishment in WDM Network:}

WDM technology has enabled wavelength routing, which provides the high-speed bandwidth transparency than electronics could provide. [9] In a wavelength-routed network, a connection between a source node and a destination node is called a light path. A light path is an optical channel that may span multiple fiber links to provide an all-optical connection between two nodes.[10] WDM multiple optical wavelengths can be used simultaneously in a fibre, which helps when trying to utilize as much of the fibre capacity as possible. Each wavelength provides a separate channel to transport data [10]. It is possible for network providers to share for instance one WDM fibre by allocating different channels for their own usage. Equipment such as OXCs or optical add drop multiplexers (OADM) can be used to connect fibres and to send and receive data on channels. Some OXCs can even connect channels between fibres. These kinds of networks make it possible for dynamic reconfiguration of the optical network topology without any physical re-wiring of fibres. A network provider can connect nodes through configuration of one or more OXCs and then release the connection to a third party as an optical point-to-point link.

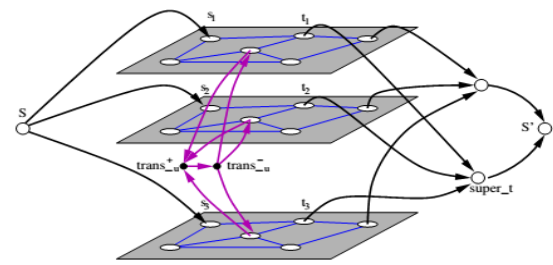

Fig1: Wavelength-routed WDM network with lightpath established between nodes.

\section{B).Routing And Wavelength Assignment:}

In order to satisfy a lightpath request in wavelength-routed WDM networks, we not only need to consider routing but the wavelength selection as well. Given a set of connection requests, the problem of setting up a lightpath by routing and assigning a wavelength to each connection is called RWA problem.[11] It can be formulated as a combinatorial problem known to be nondeterministic polynomial time (NP)-complete. Many heuristic algorithms have been proposed and evaluated under different assumptions. The routing schemes can be catalogued into static routing (including fixed-alternate routing) and dynamic routing(connection request randomly). The wavelength assignment selection policies include as follows.

1. Random assignment: For one route, a wavelength is chosen from the all-possible wavelengths set of this route randomly.

2. Most-used assignment: This scheme attempts to allocate the most utilized wavelength first. It outperforms LU and FF significantly while computation cost is similar to LU.

3. Least-used assignment: This scheme attempts to balance the load among all the wavelengths. Its performance is even worse than RWA in the evaluation so is not preferred in practice.

4. First-fit assignment: In this scheme, all wavelengths are numbered. Pick up the first available wavelength in numerical order. It does not need the global information.

\section{Twin Routing Tables Structure}

In Ant Based Routing (ABR) algorithm, a network node $\mathrm{i}$ with $\mathrm{k}_{\mathrm{i}}$ neighbors is equipped with a probabilistic pheromone table $\mathrm{R}_{\mathrm{i}}=\left[\mathrm{r}_{\mathrm{n}, \mathrm{d}}^{\mathrm{i}}\right] \mathrm{k}_{\mathrm{i}}, \mathrm{N}-1$ with $\mathrm{N}$-1rows $\left(\mathrm{N}\right.$ is the number of network nodes) and $\mathrm{k}_{\mathrm{i}}$ columns, and a P-route table with N-1rows In the pheromone table, each row corresponds to a destination node and each column corresponds to a neighbor node. The value is $\left[\mathrm{r}_{\mathrm{n}, \mathrm{d}}^{\mathrm{i}}\right.$ ]used as the selection probability of neighbor node $\mathrm{n}$ when an ant is moving toward its destination node $\mathrm{d}$. In the P-route table, each row corresponds to a destination and contains a list of $\mathrm{P}$ routes to the destination.[12] Each route is assigned a value that represents 
the goodness of this route based on its length and the number of idle wavelengths along it. The bigger the goodness of a route, the better path is considered for connection setup.

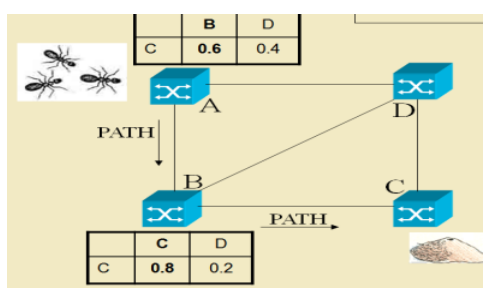

Fig: 2.Twin route table structure

\subsection{Ant's Foraging and Routing Tables Updating}

On each network node and for every t time units (second), an ant is launched with a given probability $\rho$ to a randomly selected destination; here $\rho$ and $t$ are design parameters. Each Ant is considered to be a mobile agent: it collects information on its trip, performs routing tables updating on visited nodes, and continues to move forward as illustrated in Fig. 3

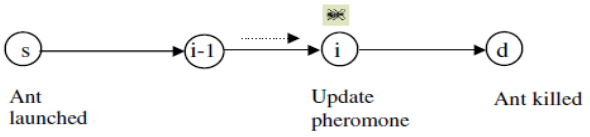

\subsubsection{Pheromone Table Updating}

Fig: 3. Ants moving and updating tasks

Whenever an ant visits a node, it updates the pheromone table element with the information gathered during its trip. Suppose an ant moves from source $s$ to destination $d$ following the path $(s \ldots i-1, i d)$. When the ant arrives at node $i$, it updates the entry corresponding to the node $\mathrm{s}$ as follows: the probability of neighbor $i-1$ is increased while the probabilities of other neighbours are decreased. If an ant visits node $i$ at time $t$, so the next values for routing entry in next time $t+1$ are determined as follows:

$$
\begin{gathered}
\mathrm{r}_{\mathrm{i}-1, \mathrm{~s}}^{\mathrm{i}}(\mathrm{t}+1)=\frac{\mathrm{r}_{\mathrm{i}-1, \mathrm{~s}}^{\mathrm{i}}(\mathrm{t})+\delta \mathrm{r}}{1+\delta \mathrm{r}} \\
\mathrm{r}_{\mathrm{n}, \mathrm{s}}^{\mathrm{i}}(\mathrm{t}+1)=\frac{\mathrm{r}_{\mathrm{n}, \mathrm{s}}^{\mathrm{i}}(\mathrm{t})+\delta \mathrm{r}}{1+\delta \mathrm{r}} \forall \mathrm{n} \neq \mathrm{i}-1
\end{gathered}
$$

Here, $\mathrm{dr}$ is the reinforcement parameter or the amount of trailing pheromone, and $\mathrm{dr}$ decreases with the length of the route and increases with the number of available wavelengths:

\section{A). P-Route Table Updating}

Besides updating the pheromone table, ant also updates the P-route table. As mentioned above, each route is associated with a goodness value that is calculated by the following formula:

$$
\mathrm{dr}=\varphi * \frac{1}{\mathrm{dl}+1} *(1+\varphi) \mathrm{dw}
$$

Here, $\boldsymbol{\varphi}$ is a scalar parameter used to adjust the emphasis between the route length and the percentage of free wavelengths. The bigger value of $\varphi$, the larger goodness value the shorter route has. The smaller value of $\varphi$, the larger goodness value the route with more idle wavelengths has. The parameter $\varphi$ should be chosen such that the shorter route has a larger goodness value, while for two routes with the same length; the route with a larger number of idle wavelengths gets a larger goodness value.

\section{B). Primary and Backup Path Computation}

Initially, in the path, a major part of the bandwidth is allocated for primary path and this part cannot be shared by other paths. Fraction of the bandwidth is allocated for backup path and this can be shared by other disjoint paths. The disjoint path can be primary or backup path. The residual bandwidth is used for future primary and backup paths computations.

Upon receiving the CNREQ, the path that has higher feasibility value and the load value lesser than ThLOAD can be selected by the destination for further processing. The backup path can be triggered in two scenarios.

(i) Failure Detection

(ii) Congestion Detection 
When any intermediate node detects node failure or fiber failure, then it sends FAILURE warning message to the source node along the alternate light path. To detect congestion, the Value of path load should not exceed the ThLOAD. If the load value exceeds beyond ThLOAD, then it will lead the network to congestion and consequently to the blocking of services. Thus, to prevent these, we let the intermediate node to send a CONGESTION warning message to the source before it exceeds ThLOAD.

If the source node receives either FAILURE or CONGESTION warning message from the intermediate nodes, it triggers the backup path protection and establishes back up light path from the estimated set of light paths (from the ant agents). The source node looks its pheromone and load table and select the path that has low load value and higher feasibility value as next hop and precedes the transmission. During the transmission, if the load value exceeds the ThLOAD, then backup path can be triggered by the corresponding node.

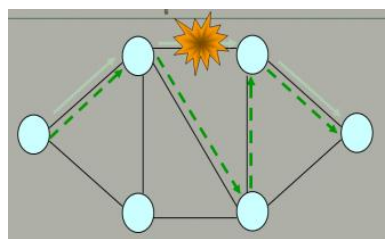

Fig: 3.(a) Primary path routing

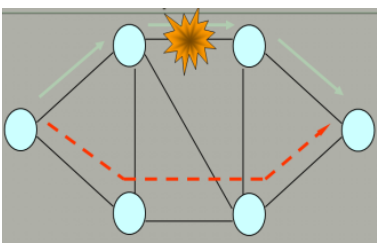

(b) Backup path routing

\section{C).Multilayer-graph model}

Instead of separated processing of the routing and wavelength assignment sub-problems, we consider the dynamic RWA problem as a whole. Thus, a new model called the multilayer-graph model is put forward. Based on it, route selecting and wavelength assignment is completed simultaneously using the previous mentioned routing strategies for light-path requests Based on the multilayer-graph model in [11], a lightpath must occupy the same wavelength on all links of the path used, a property known as the wavelength continuity constraint. Previous work has shown that wavelength conversion can considerably improve the performance in all optical networks. But it will cost much if all nodes in the WDM network have wavelength conversion. So we consider that part of the nodes have wavelength conversion. In this paper, we present an extended multilayergraph model called the interconnected multilayer-graph model (IMG) [11].

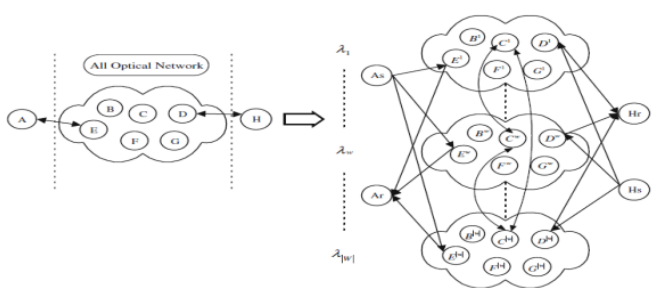

Fig: 4. Inter connected multilayer network mode

\section{Connection Setup}

Upon the arrival of a connection request, the $\mathrm{k}$ best routes that have the biggest goodness values in the P-route table are selected as the routes candidates for light path setup. The light path setup is similar to other alternate routing methods: the source node sends in parallel some needle packets along these $\mathrm{k}$ routes toward destination to request a path setup. [13]At the destination node, the route with the highest goodness value is selected for connection setup. If no free wavelength is available for all these $\mathrm{k}$ routes, the connection request will be blocked. We use in our algorithm two alternate routes $(\mathrm{k}=2)$ as suggested in that alternate routing methods using too many alternate routes do not significantly improve network performance.

\section{The pseudo-code of the main steps in our algorithm can be summarized as follows:}

\section{\{Ant generation\}}

Do

For each node in network Connection requests

Select a random destination;

Launch ants to this destination

With a probability $\rho$;

Constant Bit Rate transmission through lightpath

End for 
Increase time by a time-step for ants' generation;

Until (End of simulation)

\section{\{Ant foraging\}}

For each ant from source $\mathrm{s}$ to destination $\mathrm{d}$ do (in parallel)

While current node $\mathrm{i}\langle>\mathrm{d}$

Update pheromone table elements;

Update P-route table;

Push trip's state into stack;

If (found a next hop)

Move ant to next hop ;

Else

Kill ant;

End if

End while

End for

\{Routing and Wavelength Assignment

For each connection request do (in parallel)

Select a path based on first-highest-probability lookup

Select the first available wavelength on path

If (found)

Setup a lightpath

Else

Select another path based on second highest probability

Select the first available wavelength on path

If (found)

Setup a lightpath

Else

Consider a blocking case

End if

End if

End for

\{Primary and backup path computation\}

For each node in network Connection requests

If the request is high priority traffic then;

If primary path is found;

Record route information and wavelength assignment;

Update network state and link cost function;

If the request is low priority traffic then;

If backup pathis found;

Record route information and wavelength assignment;

Update network state and link cost function;

\section{\{Computing Working path\}}

If working path found;

Record route information and wavelength assignment;

Update network state and link cost function;

If working path failed;

Primary path and backup path are computed;

End if;

\section{Simulation Results Analysis}

In all the simulation cases, we assume the arrival traffic follows a Poisson process and the service time is exponential distributed with mean second. The rates in the simulation of numerical results are expressed in requests/sec... Each node has the same probability to send packet to the rest of nodes. [14] In design optical WDM network with nodes 15 and links 19 having wavelength $8 \mathrm{~nm}$, and average time holding is $10 \mathrm{sec}$, where blocking probability is about $5 \%$.after network established we place ants in network to search best route where time generation of ants is $\mathrm{t}=1 \mathrm{~ms}$ and delay is $10 \mathrm{~ms}$.Ants in the network randomly searches for route and finally 
finds shortest paths and start updating P-route table and phermenon table which is known as lightpath reference table updating. By using First-Fit heuristic algorithms wavelength assignment is done to WDM networks to overcome blocking probabilities, and this improvement becomes more significant for network with larger wavelengths per link. This wavelength assignment blocking probability is verified with CBR and in Exponential terms. After connection request arrives ants in the networks randomly search for best path for every node to node, after finding best short path light paths are established. The delay time, utilization of ants and throughput is updated in phermenon table. After connection request failure occurred at any node or link by using primary and backup path computation mechanism it is solved. In case of data is transmitted through working path if failure occurred immediately data is transmitted though the primary path which is shared by other node links, if primary path is failed alternate backup path is routed in network in advance from source node to destination node. In Multilayer network after wavelength assignment data is transfer through one network to another network by inter linking nodes in networks. Similarly we compare our results with other previous algorithms to show better profits.

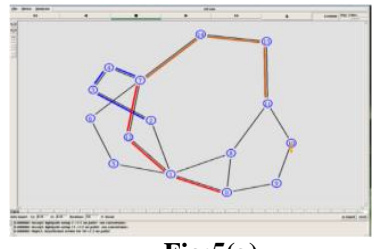

Fig:5(a)

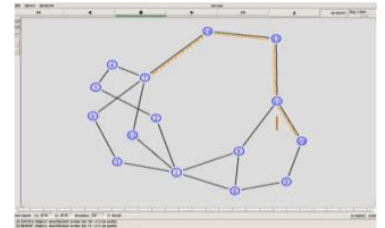

Fig:5(b)

Fig: 5.(a) Establishing Lightpath for WDM networks Fig:5(b).constant bit rate transmission and updating routing tables

A possible reason for this is that we do not use the reduced traffic model. Finally, we see that the results are not sensitive to the number of wavelength and our numerical approach remains accurate even with a relative large number of wavelengths. In this section, [17]we verify the performance of our ABR (Ant Based Routing) algorithm based on simulation in a circuit-switched routing module for WDM network in the ns-2 Network Simulator. For comparison, we also conduct simulation for the ASP and FPLC algorithms that adopt two alternate routes.

\section{Lightpath \& Wavelength assignment WDM Network}

After connection request arrives ants in the networks randomly search for best path for every node to node, after finding best short path light paths are established and p-route table is updated by mobile agents called ants. Packet transmission is done while wavelength $\mathrm{W}=8 \mathrm{~nm}$ allotted through the network, so data is travelled through the network constantly while updating the phermenon table. The delay time, utilization of ants and throughput is updated in phermenon table similarly we compare our results with other previous algorithms to show better profits.

\section{Pheromone Table Updating for best light paths in WDM networks}

A). P-route table updating of optimal lightpath in constant Bit Rate (CBR).

\begin{tabular}{|c|c|c|c|c|}
\hline $\begin{array}{c}\text { Source/Desti } \\
\text { nation }\end{array}$ & Possible path & $\begin{array}{c}\text { Path } \\
\text { ref no }\end{array}$ & $\begin{array}{c}\text { Consta } \\
\text { nt Bit } \\
\text { Rate }\end{array}$ & $\begin{array}{c}\text { Optimum } \\
\text { path }\end{array}$ \\
\hline $2-7$ & $2-3-4-7$ & 1 & $10^{-3}$ & \\
& $2-1-12-7$ & 2 & $10^{-7}$ & $2-3-4-7$ \\
& $2-1-5-6-7$ & 3 & $10^{-13}$ & \\
\hline $7-11$ & $7-4-3-2-1-8-$ & 1 & $10^{-13}$ & \\
& 11 & 2 & $10^{-3}$ & $2-1-0-9-10$ \\
& $7-14-13-11$ & 3 & $10^{-10}$ & \\
\hline $0-7$ & $7-12-1-8-11$ & & & \\
& $0-1-12-7$ & 1 & $10^{-3}$ & \\
& $0-8-1-12-7$ & 2 & $10^{-13}$ & $7-14-13-11-$ \\
& $0-8-1-2-3-4-7$ & 3 & $10^{-17}$ & 10 \\
\hline
\end{tabular}


B). Pheromone Table Updating

\begin{tabular}{|c|c|c|c|c|c|}
\hline $\begin{array}{c}\text { Source- } \\
\text { Destination }\end{array}$ & $\begin{array}{c}\text { Path } \\
\text { Reference }\end{array}$ & $\begin{array}{c}\text { Constant } \\
\text { Bit Rate }\end{array}$ & Request time & $\begin{array}{l}\text { Over all N/w } \\
\text { Delay time }\end{array}$ & Dropped Time \\
\hline $2-7$ & $1(2-3)$ & $39884^{*} 10^{-3}$ & $23.912121 \mu \mathrm{se}$ & & \\
$(2-3-4-7)$ & $2(3-4)$ & $39757 * 10^{-3}$ & $23.910143 \mu \mathrm{se}$ & $2.05577 \mu \mathrm{sec}$ & $30.76588 \mu \mathrm{se}$ \\
& $3(4-7)$ & $39900 * 10^{-3}$ & $23.93635 \mu \mathrm{se}$ & & \\
& $(2-3-4-7)$ & $45675 * 10^{-3}$ & $25.96789 \mu \mathrm{se}$ & & \\
\hline $7-11$ & $1(7-14)$ & $34968 * 10^{-3}$ & $24.116512 \mu \mathrm{se}$ & & $32.97682 \mu \mathrm{se}$ \\
$(7-14-13-11)$ & $2(14-13)$ & $36987 * 10^{-3}$ & $23.659856 \mu \mathrm{se}$ & $3.873253 \mu \mathrm{sec}$ & \\
& $3(0-7)$ & $41500^{*} 10^{-3}$ & $26.798975 \mu \mathrm{se}$ & & \\
& $(7-14-13-11)$ & $48976^{*} 10^{-3}$ & $27.989765 \mu \mathrm{se}$ & & \\
& $1(0-1)$ & $25876^{*} 10^{-3}$ & $22.66523 \mu \mathrm{se}$ & & \\
$(0-1-12-7)$ & $2(1-12)$ & $32987 * 10^{-3}$ & $25.910143 \mu \mathrm{se}$ & $7.147139 \mu \mathrm{sec}$ & $39.76488 \mu \mathrm{se}$ \\
& $3(12-7)$ & $39900 * 10^{-3}$ & $27.936135 \mu \mathrm{se}$ & & \\
& $(0-1-12-7)$ & $45998 * 10^{-3}$ & $29.812369 \mu \mathrm{se}$ & & \\
\hline \multicolumn{7}{|r}{}
\end{tabular}

\section{B). Pheromone Table Updating}

In above table Pheromone table updated by ANT selecting best light path in WDM network at $50 \mathrm{Mb}$ transmission in network at Constant Bit Rate, request time, delay time, and drop time are updated.
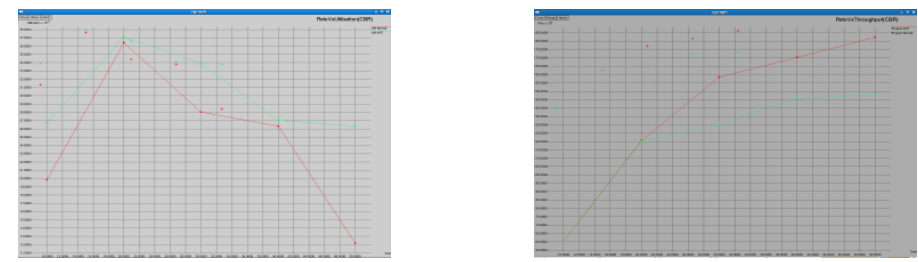

Fig:6(a) Rate vs Utilization in (CBR) Fig6(b) Rate vs Throughput in (CBR)

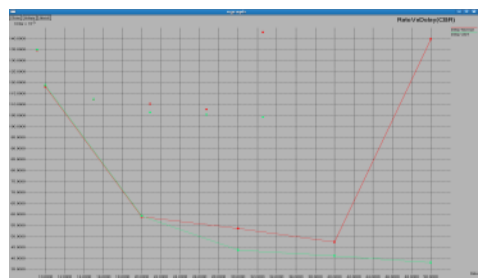

Fig: 6 (c). Transmission Rate Vs Delay (CBR)

In the above specified simulation graphs are executed in terms of Constant Bit Rate. Ant network performs best results than normal network as Utilization of mobile agents is high rate of network is high and it is measured in throughput of two network, as rate of traffic is high delay of Ant network is less as compared to normal network.
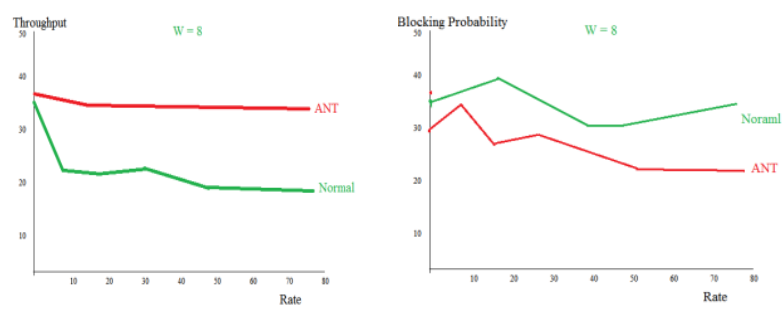

Fig: 7. (a) Rate Vs Wavelength at $8 \mathrm{~nm}$ Ant \& Normal networks(Expo)

Fig: 7 (b) Rate vs Blocking Probability at wavelength=8nm in (Expo)

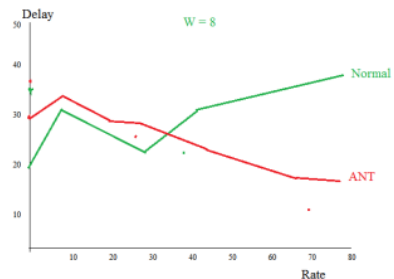

Fig:7(c).Rate vs Delay at Wavelength $=8 \mathrm{~nm}$ in $($ Expo $)$ 


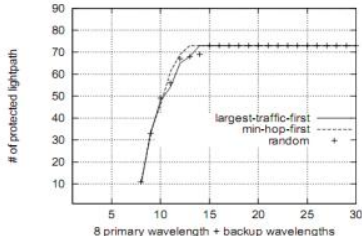

Figure 5. The number of protected lightpets

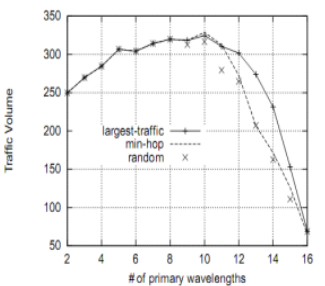

Figure 8 (a). The number of protected lightpaths

Figure 8 (b). Total volume of the traffic protected by backup lightpaths before IP routing table update

From figures The shared link protection mechanism is considered for improving wavelength utilization under the assumption that the WDM network is highly reliable and the failure seldom occurs. Our objective is therefore to minimize the number of utilized wavelengths on the link.
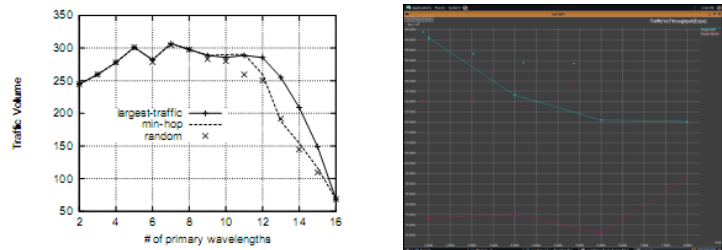

Figure 9(a). Total volume of the traffic protected by backup lightpaths after IP routing table update.

Fig9 (b)Traffic vs throughput(Expo)

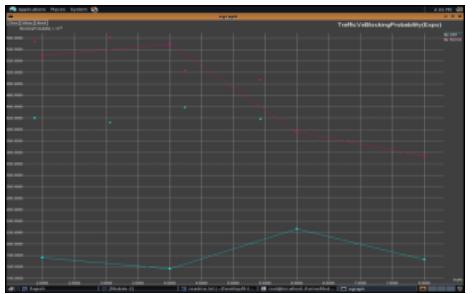

Fig:9 (c) Traffic vs Blocking Probability (Expo)

By simulation results it shown that inter connected Multilayer survivable routing out performs best results in transmission of data through the lightpaths. We use the First-Fit algorithm, which is a heuristic algorithm proposed. The First-fit algorithm works as follows. First, it places the lightpath between nodes if there exists a fiber. Then, attempts to place lightpaths between nodes in the order of descending traffic rate are made. Finally, if there still exist non utilized wavelengths, lightpaths are placed as much as possible utilizing those wavelengths.

1) While the First-fit algorithm places lightpaths even if the lightpath has already been placed, we do not set up multiple Wavelengths between two nodes so that remaining wavelength are left as a possible use for the backup lightpaths.

2) While the First-fit algorithm places lightpaths randomly if there exist unused wavelengths at the final step of the algorithm, we do not assign non-utilized wavelengths due to the same reason above.

It is ideal that the WDM network could protect all the lightpaths so that the traffic on the primary lightpath can be switched to the backup lightpath in the order of $10 \mathrm{msec}$. However, we need to consider the tradeoff relationship between the processing capability of IP routers and a limitation on the number of wavelengths. Setting up more backup lightpaths can protect more lightpaths. However, because of the limitation on the number of wavelengths, the number of primary lightpaths should be limited in order to increase the number of backup lightpaths. The smaller number of primary lightpaths results in that the traffic load at the IP router is increased, and that bottleneck caused by the IP router cannot be resolved. On the contrary, we can expect that more traffic can be carried by primary lightpaths if the wavelengths used for the primary lightpaths is increased, but in that case, the advantage of protection mechanisms of the WDM network cannot be enjoyed.

There is another problem. While a WDM protection mechanism switches to the backup lightpath in order of $10 \mathrm{msec}$, IP router may change the route to better one after IP routing table is updated. Suppose that after the failure occurs, the lightpath 'ij' utilizing wavelength $\mathrm{k}$ is switched to the backup lightpath, which results in the increasing of propagation delay by its nature. After IP updates its routing table (typically in the 
order of $10 \mathrm{sec}$ after), the IP router may find route (which may consist of two or more concatenated lightpaths) shorter than the backup lightpath prepared by the WDM protection mechanism.

(1) In Step. 1, after selecting a lightpath hij, set $\{\mathrm{S}\}$, elements of which are node pairs utilizing lightpath hij.

(2) Calculate the increased delay $\theta$ under the assumption that the backup lightpath is placed.

(3) For every node pair s-d in $\{\mathrm{S}\}$, calculate the delay of primary lightpath d-s-d and that of the second shortest path s-d. Then, check whether the sum of s-d and $\theta$ exceeds the delay of the second shortest path or not. If it is true, check the next lightpath hkij without protecting the current lightpath hk ij. It is difficult to identify how many wavelengths should be assigned for primary and backup lightpaths, since it depends on the requirement of the network capacity provided by the primary lightpaths and the network survivability by the protection mechanism of the WDM network.

\section{Conclusion}

In this paper, we have investigated the lightpath optimization survivability routing in WDM networks. As Survivable Routing have considered the reliability mechanism in the WDM network. By assuming Virtual topology network the lightpath establishment is done by using Ant Colony optimization Schemes and shown updating of lightpaths in P-route table and Phermenon table by using mobile agents called Ant's. By assuming single-failure within the network, we have formulated the shared link protection mechanism as an optimization problem. It is formulated as Multi layer interconnected Problem, and computationally intensive as the network size grows. Accordingly, we have proposed the heuristic approaches and compared those with the solution obtained by MILP. Through numerical examples, we have compared the required number of wavelengths for the reliable network. By considering the functional partitioning of IP routing and WDM protection for reliable networks with our First-fit heuristic algorithm, discussed the effect of interaction between IP and WDM layers. The results have shown that the largest-traffic-first approach is best if our primary concern is traffic load at the IP router after the failure. Our heuristic approaches for the reliable networks do not explicitly formulate the minimization of the required number of wavelengths to construct the logical topology. The proposed First-Fit algorithm, more effective one is necessary so that in multi-layer survivability, a few node pairs did not use the backup lightpath prepared by WDM protection of our algorithms after routing tables are updated.

\section{Bibliography}

[1]. Son-Hong Ngo, Xiaohong Jiang, Susumu Horiguchi, and Minyi Guo: Dynamic Routing and Wavelength Assignment in WDM Networks with Ant-Based Agents. L.T. Yang et al. (Eds.): EUC 2004, LNCS 3207, pp. 829-838, 2004.

[2]. Ramaswami, R, Sivarajan, K.N.: Routing and wavelength assignment in all-optical net-works. IEEE/ACM Transactions on Networking, vol. 3 (1995) 489-500.

[3]. B. Tonderayi Mangara and F. Wilhelm Leuschner,'Survivable Routing for WDM Networks: Challenges faced by the South African Service Providers", In Proceedings of the Southern African Telecommunications Networks and Applications Conference (SATNAC), 2003.

[4]. S. Ramamurthy, Laxman Sahasrabuddhe, and Biswanath Mukherjee, "Survivable WDM Mesh Networks", IEEE Journal of Lightwave Technology, 2003.

[5]. H. Zang, J. Jue, and B. Mukherjee, "A Review of Routing and Wavelength Assignment Approaches for Wavelength Routed Optical WDM Networks.” Optical Networks Magazine, pp. 47 - 60, January 2000.

[6]. M. Tornatore, G. Maier, and A. Pattavina, "WDM Network Design by ILP Models Based on Flow Aggregation," IEEE/ACM Transactions on Networking, Vol 15, No 3, pp 709 - 720, 2007.

[7]. B. Caenegem, W. Parys, F. Turck, and P. Deemeseter, "Dimensioning of Survivable WDM Networks," IEEE Journal on Selected Areas of Communication, pp. 1146 - 1157, September 1998.

[8]. D. Banerjee and B. Mukherjee, "Wavelength9routed Optical Networks: Linear formulation, resource budgeting tradeoffs and a reconfiguration study.” IEEE/ACM Transactions on Networking, pp. 598-607, October 2000.

[9]. D. Banerjee and B. Mukherjee, "A practical approach for routing and wavelength assignment in large wavelength9routed optical networks," IEEE Journal on Selected Areas of Communication, pp. 903 - 908, June 1996.

[10]. S. Even, A. Itai, and A. Shamir, "On the Complexity of Timetable and Multicommodity Flow Problems," SIAM Journal of Computing, Vol. 5, pp. 6919703, 1976.

[11]. A. McGregor and B. Shepherd. "Island Hopping and Path Coloring with Applications to WDM Network Design," SIAM Symposium on Discrete Algorithms, pp. 864 - 873, 2007.

[12]. J. Suurballe, "Disjoint Paths in a Network," Networks, Vol 4, pp 125 - 145, 1974.

[13]. S. Ramamurthy, "Optical Design of WDM Network Architectures," Ph.D. Dissertation, University of California, Davis, 1998.

[14]. Vinh Trong Lea, Xiaohong Jiangb, Son Hong Ngoa, Susumu Horiguchib and Yasushi Inoguchic, “A novel dynamic survivable routing in WDM optical networks with/without sparse wavelength conversion", ELSEVIER, Optimal Switching and Networking, 2006.

[15]. Daniel Dao-Jun Kan, Aradhana Narula-Tam and Eytan Modiano, "Lightpath Routing and Capacity Assignment for Survivable IPover-WDM Networks", Proceedings of the IEEE 7th International Workshop on Design of Reliable Communication Networks, (DRCN), 2009.

[16]. Eytan Modiano and Aradhana Narula-Tam, "Survivable routing of logical topologies in WDM networks", Proceedings of Twentieth Annual Joint Conference of the IEEE Computer and Communications Societies, IEEE INFOCOM 2001. 
Light Path Optimization Implementation for Survivable Routing and Wavelength Assignment .....

[17]. Debasis Mandal, Satyajit Nath and Bivas Mitra, "Survivable Routing in WDM Weighted Networks", Proceedings of the $2^{\text {nd }}$ IEEE International Conference on Communication System Software and Middleware (COMSWARE), 2007.

[18]. ] Kayi Lee and Eytan Modiano, "Cross-Layer Survivability inWDM Networks with Multiple Failures", In Optical Fiber Communication Conference and Exposition and The National Fiber Optic Engineers Conference, OSA Technical Digest (CD) (Optical Society of America), 2008.

[19]. Timothy Hahn, Shen Wan Montana State University: An Ant Colony Optimization Implementation for Dynamic Routing and Wavelength Assignment in Optical Networks.

[20]. Z. Na and S. Jaijin, "A Distributed Strategy for Dynamic Routing and Wavelength Assignment in ASON Network," International Conference on Transparent Optical Networks, pp. 201 - 204, 2005. 